\title{
Transverse Differential Pressure Flowmeter ${ }^{\dagger}$
}

\author{
Ippei TORIGOE*
}

\begin{abstract}
A new method is proposed for measuring the pulsating flow rate. Under pulsating conditions the output of conventional differential pressure flowmeters contains the acceleration term due to the fluid inertia, which causes a pulsation error in the measurement of the flow rate. In the proposed method, the stream to be measured is divided into two branch streams and each stream is constricted by the converging section of each branch channel. The pressure difference between the two channels is detected by a manometer at a point where the acceleration term of each stream cancels out. The instantaneous flow rate can then be obtained from the output of the manometer without being affected by the acceleration term. An experimental apparatus was built, and several experiments for air flow were performed with this apparatus. The results indicate that the proposed flowmeter is capable of measuring the instantaneous flow rate in the pulsating regime.
\end{abstract}

Key Words : pulsating flow, instantaneous flow rate, differential pressure flowmeter, acceleration error, branch stream

\section{Introduction}

The problem of pulsating flow measurement has been an object of study for a long time. The difficulties encountered in metering pulsating flow were first reported 90 .years ago by Venable ${ }^{1)}$. As early as 1955, Oppenheim and Chilton published a survey of the extensive literature on the problem ${ }^{2}$. In spite of all the investigations, the problem still exists and no complete solution has been established ${ }^{3)}$. Broadly speaking, the earlier investigations can be grouped into two categories by objective: (1) to attenuate the pulsation before they reach the flowmeter; and (2) to evaluate the correction factors which can be applied to the indicated flow rate reading of existing types of flowmeters. In recent decades there has been renewal of interest in the measurement of pulsating flow ${ }^{4) \sim 8}$. While the majority of earlier investigations were concerned with the measurement of the time-averaged rate of pulsating flow, increasing attention has been directed to the instantaneous flow rate in recent papers.

$\dagger$ Presented 32nd SICE Annual Conference $(1993 \cdot 8)$

* Computer Center, Kumamoto University, 2-39-1,

Kurokami, Kumamoto

(Received November 14, 1994)

(Revised April 7, 1995)
Differential pressure flowmeters are the most common device to be found in industry, and have also been the major object of the investigations on pulsating flow measurement. In this sort of flowmeter the pressure difference is detected by a manometer between two pressure taps located along the stream. For steady flow, the output of the manometer is proportional to the square of the flow rate. Under pulsating conditions, in contrast, the output contains the acceleration term due to the inertia of the fluid enclosed by the conduit boundary and the two tap sections. This term causes a pulsation error, "the acceleration error", in the flow rate measurement even after "manometer errors" and so-called "square root errors" have been eliminated. In the last few decades, a few attempts have been made to eliminate the acceleration error of differential pressure flowmeters $^{9) \sim 11)}$.

The aim of this paper is to advance a new flowmeter, named Transverse Differential Pressure Flowmeter (TDPF) for the present, to measure the instantaneous rate of pulsating flow. In the TDPF, the stream to be measured is divided into two branch streams and each stream is constricted by the converging section of the branch channels. Two pressure taps, one tap in each branch channel, are made at a point where the acceleration term of each stream 
cancels out. The pressure difference between these taps lying in the transverse direction to the stream is detected by a manometer. Since this pressure difference no longer contains the acceleration term, the normal steady flow relationship holds instantaneously between the output of the manometer and the flow rate. The instantaneous rate of flow can then be obtained from the output of the manometer without being affected by the fluid inertia.

\section{Principle}

\subsection{Differential Pressure Flowmeters Under Pulsating Conditions}

We will begin by considering conventional differential pressure flowmeters under pulsating conditions. The system diagram of a venturi meter, given as an example, is shown in Fig. 1. If the contraction of the meter is gradual enough and the viscosity of the fluid can be neglected, we can assume that the flow is one-dimensional. One-dimensional inviscid flow is described by the continuity equation

$$
\frac{\partial \rho}{\partial t}+\frac{\partial}{\partial x}(\rho S U)=0
$$

and the equation of motion

$$
\rho \frac{\partial U}{\partial t}+\rho U-\frac{\partial U}{\partial x}+\frac{\partial p}{\partial x}=0
$$

where $\rho$ is the fluid density, $S$ is the cross-sectional area of the conduit, $U$ is the flow velocity, and $p$ is the pressure. Integrating ( 2 ) along the stream from $x=$ 0 to $x=L$, we have at a given instant

$$
\begin{aligned}
& \rho \int_{0}^{L} \frac{\partial U}{\partial t} d x+\frac{\rho}{2}\left\{U^{2}(L, t)-U^{2}(0, t)\right\} \\
& \quad+p(L, t)-p(0, t)=0
\end{aligned}
$$

For incompressible fluids, the continuity equation (1) can be expressed in the form of the continuity of the volumetric flow rate $Q$;

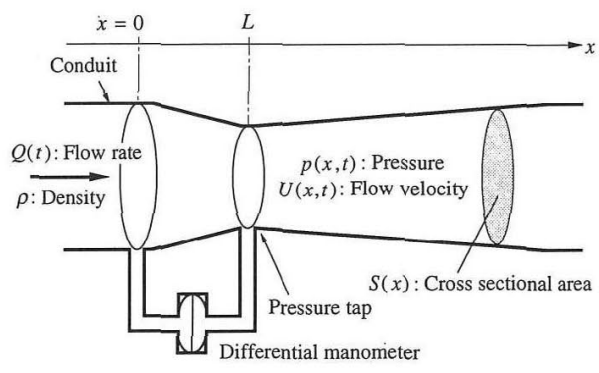

Fig. 1 Venturi meter under pulsating conditions

$$
S(x) U(x, t)=Q(t)
$$

The substitution (4) into (3) yields

$$
\begin{aligned}
& \rho \frac{\partial Q}{\partial t} \frac{1}{S(0)} \int_{0}^{L} \frac{S(0)}{S(x)} d x+\frac{1}{2} \rho Q^{2}\left\{\frac{1}{S^{2}(L)}-\frac{1}{S^{2}(0)}\right\} \\
& \quad+p(L, t)-p(0, t)=0
\end{aligned}
$$

If the flow is steady, equation (5) reduces to Bernoulli's equation

$$
p(0)-p(L)=\frac{1}{2} \rho Q^{2}\left\{\frac{1}{S^{2}(L)}-\frac{1}{S^{2}(0)}\right\}
$$

Using this equation we can measure the flow rate by detecting the pressure difference between $x=0$ and $x$ $=L$. Under pulsating conditions, in contrast to steady flow, the first term in the left hand side of equation (5) does not vanish. The pressure difference thus contains a term proportional to the time derivative of the flow rate in addition to the steady flow term. Owing to this additional term, the flow rate can not be obtained by the same method as that for steady flow. The points made so far apply in principle for any other meter of the differential pressure type such as the orifice meter and the nozzle.

\section{2 Transverse Differential Pressure Flowmeter}

Let us now turn to the real subject. The principle of the Transverse Differential Pressure Flowmeter (TDPF) is schematically depicted in Fig. 2. Throughout this section we assume the fluid is incompressible and inviscid - the validity of this assumption is taken up in the next section. We also assume that the conduit is uniform except for the branch section and the number of branch channels is two; these are for the sake of simplicity and are not theoretically necessary. The flow through the main conduit - that is assumed to have a uniform profile - is

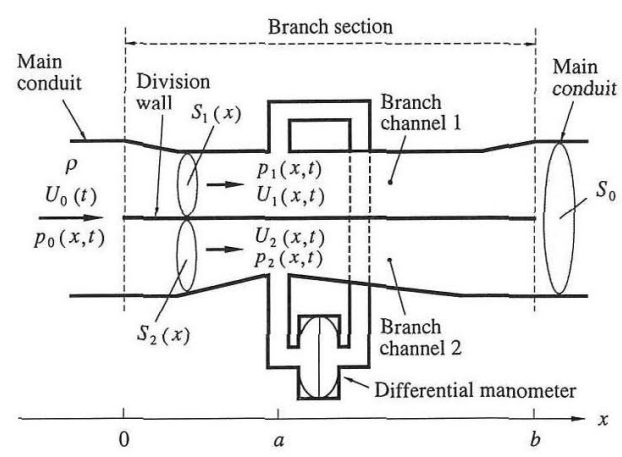

Fig. 2 Principle of the Transverse Differential Pressure Flowmeter 
divided at $x=0$ into two branch streams by the division wall, and they join each other at the tail of the wall $(x=b)$. Each branch stream is first accelerated by the converging section of the channel and then decelerated gradually by the diverging section. The pressure difference between the two channels is detected at $x=a$. The point of the TDPF is its geometry; the branch channels are made in such a manner that the following four formulae hold:

$$
\begin{aligned}
& \frac{S_{1}(0)}{S_{1}(b)}=\frac{S_{2}(0)}{S_{2}(b)} \\
& \int_{0}^{b} \frac{S_{1}(0)}{S_{1}(x)} d x=\int_{0}^{b} \frac{S_{2}(0)}{S_{2}(x)} d x \\
& \int_{0}^{a} \frac{S_{1}(0)}{S_{1}(x)} d x=\int_{0}^{a} \frac{S_{2}(0)}{S_{2}(x)} d x \\
& \frac{S_{1}(0)}{S_{1}(a)} \neq \frac{S_{2}(0)}{S_{2}(a)}
\end{aligned}
$$

where $S_{i}$ is the cross-sectional area, and the subscript 1 and 2 denote the quantity concerns respectively the branch channel 1 and 2. From the continuity of the volumetric flow rate and equation ( 7 ), the velocity of each stream at the channel outlet is equal to that at the inlet;

$$
\begin{aligned}
& U_{1}(0, t)=U_{1}(b, t)=U_{0}(t), \\
& U_{2}(0, t)=U_{2}(b, t)=U_{0}(t)
\end{aligned}
$$

where $U_{1}, U_{2}$ is the flow velocity in the branch channel, $U_{0}$ is the flow velocity at $x=0$, the subscript 0 denotes the quantity concerns the main conduit. Integrating the equation of motion of each stream over the branch channel section, we have

$$
\begin{gathered}
\rho \int_{0}^{b} \frac{\partial U_{i}}{\partial t} d x+\frac{\rho}{2}\left\{U_{i}^{2}(b, t)-U_{i}^{2}(0, t)\right\} \\
+p_{i}(b, t)-p_{i}(0, t)=0, i=1,2
\end{gathered}
$$

where $p_{1}$ and $p_{2}$ are the pressures in the branch channel. On the basis of this equation we can evaluate the pressure at the outlet of the branch channels. Using (11) and $U_{i}(x, t) S_{i}(x)=U_{i}(0, t) S_{i}(0) \quad(i=0,1,2)$, we have

$$
\begin{aligned}
& p_{0}(0, t)-p_{1}(b, t)=\rho \frac{\partial U_{0}}{\partial t} \cdot \int_{0}^{b} \frac{S_{1}(0)}{S_{1}(x)} d x \\
& p_{0}(0, t)-p_{2}(b, t)=\rho \frac{\partial U_{0}}{\partial t} \cdot \int_{0}^{b} \frac{S_{2}(0)}{S_{2}(x)} d x
\end{aligned}
$$

where $p_{0}$ is the pressure in the main conduit. The substitution of ( 8 ) into (13) leads to

$$
p_{1}(b, t)=p_{2}(b, t)
$$

Equations (11) and (14) indicate the following; a flow which has a uniform profile of velocity and pressure at the inlet of the branch section $(x=0)$ discharges into the downstream conduit with a uniform profile. In this case the volumetric flow rate through the branch channels is proportional to the cross-sectional area of the respective channel inlet. Unless ( 7 ) and ( 8 ) hold, it is not certain that a flow discharges into the downstream conduit at a uniform velocity even if it has a uniform velocity profile in the upper stream. Namely, equations ( 7 ) and (8) are the sufficient conditions for the uniformity of the flow velocity within the main conduit. It may be worth mentioning, in passing, that the term

$$
\int_{0}^{b} \frac{S_{i}(0)}{S_{i}(x)} d x
$$

has a dimension of length and is interpreted to express the effective length of each branch channel. Equation ( 8 ) is considered to be a representation of the condition that the two branch channels are equal in their effective length.

Let us next consider the pressure at the taps. Integrating the equation of motion of each stream from the inlet to the tap section, we have the following equations

$$
\begin{aligned}
& p_{0}(0, t)-p_{1}(a, t)=\frac{1}{2} \rho Q_{1}^{2}\left(\frac{1}{S_{1}^{2}(a)}-\frac{1}{S_{1}^{2}(0)}\right) \\
& \quad+\rho \frac{\partial U_{0}}{\partial t} \cdot \int_{0}^{a} \frac{S_{1}(0)}{S_{1}(x)} d x \\
& p_{0}(0, t)-p_{2}(a, t)=\frac{1}{2} \rho Q_{2}^{2}\left(\frac{1}{S_{2}^{2}(a)}-\frac{1}{S_{2}^{2}(0)}\right) \\
& \quad+\rho \frac{\partial U_{0}}{\partial t} \cdot \int_{0}^{a} \frac{S_{2}(0)}{S_{2}(x)} d x
\end{aligned}
$$

where $Q_{1}$ and $Q_{2}$ are the volumetric flow rates through the respective branch channels. Substituting ( 9 ) together with $Q_{i}(t) / S_{i}(0)=Q_{0}(t) / S_{0}$ into (15), we get the pressure difference between the two branch channels ;

$$
\begin{aligned}
& p_{1}(a, t)-p_{2}(a, t) \\
& \quad=\frac{1}{2} \rho Q_{0}^{2}(t) \cdot \frac{1}{S_{0}^{2}} \cdot\left(\frac{S_{2}^{2}(0)}{S_{2}^{2}(a)}-\frac{S_{1}^{2}(0)}{S_{1}^{2}(a)}\right)
\end{aligned}
$$

where $S_{0}$ is the cross-sectional area of the main conduit. Equation ( 9 ) indicates that the two branch channels are equal in effective distance between the inlet and the pressure tap. Therefore the acceleration terms, the second terms in the right hand side of (15), cancel each other. Further, since the branch channels are made in such a way that the right hand side of (16) does not vanish, that is, inequality (10) holds, the flow rate through the conduit, $Q_{0}$, can be measured from the pressure difference at $x=a$. 


\section{3 Influence Quantities}

The principle described in the preceding section is based on flow of an inviscid and incompressible fluid with a uniform velocity profile. Departures in real flow from these conditions - the influence quantities - can affect the flow rate measurement by the TDPF. The errors associated with a particular influence quantity depend on the construction and configuration of individual embodiments. To discuss the influence quantities as a whole is thus beyond the scope of this paper. Here, we will mention the subject only summarily.

\section{(1) Compressibility}

The flow velocity is assumed to pulsate in phase over the branch channels. Strictly speaking, this is only the case if the fluid is incompressible and the channel walls are rigid. However, if the pulsation wavelength in the fluid is much longer than the dimensions of the branch channels, that is,

$$
b \ll \frac{c}{f}
$$

where $c$ is the sound speed and $f$ is the pulsation frequency, we can consider the assumption of uniform pulsation to apply approximately. As to the errors due to the fluid expansion, we will have to introduce an expansion factor to correct them. The factor may be derived from thermodynamic considerations for those channels which have a gradual contraction, or it may be determined empirically in the case of the channels with an abrupt contraction. Anyhow, the determination of the expansion factor is the subject for a future study.

(2) Viscosity

In the measurement with conventional differential pressure flowmeters, the discharge coefficient is introduced to take into account the velocity profile, the energy loss and the contraction geometry. For the venturi meters, where the contraction is gradual, the agreement is within 1 to 3 percent between the true flow rate and the theoretically calculated value, and the discharge coefficient is close to unity. Thus we can expect also for the TDPF that the pressure difference calculated theoretically is in good agreement with the true value if the contraction of the branch channels is gradual. Furthermore, owing to its differential structure, it is likely that the viscous pressure drop of each channel between the inlet and the pressure tap will also cancel out. For the TDPF with a gradual contraction, the agreement of the theoretical value with the true value may be better than the venturi meters. When the contraction of the branch channels is abrupt, on the other hand, we will have to introduce an empirically determined correction factor.

\section{(3) Velocity profile distortion}

The principle of the TDPF is founded on the assumption that the flow rate through each branch channel is proportional to the inlet cross-sectional area. Profile distortion can therefore cause a serious error and may be the most important influence quantity of the TDPF. If there are those piping elements in the upper stream which distort the profile, a sufficient straight length of pipe or, for shorter length, a flow conditioner should be installed to restore an acceptable velocity profile. Further, the element within the branch channel itself - such as the division wall - can distort the profile. The branch channel must be designed with due regard to this point and be manufactured carefully.

\section{4 Possible Embodiments}

In Fig. 2, the flow is divided by the straight division wall and each branch stream is constricted by the converging wall of the conduit. This is one possible embodiment of the TDPF. Fig. 3(a) illustrates schematically another possible embodiment; in which a pipe of a uniform cross-sectional area forms one branch channel, and an orifice plate is employed as a contraction element in the other branch channel. The pressure difference is detected between the two branch channels instead of across the orifice plate. Fig. 3(b) shows another modification of the TDPF. A tube, the thickness of which varies along its axis, is placed concentrically with the main conduit. This concentric inner tube functions as a contraction element as well as a division wall. The pressure difference is detected across the inner tube wall. It goes without saying that in any embodiments the branch channels should be made in such a manner that the formulae ( 7 ) to (10) hold.

Randall proposed a double-tube type sensor which resembles in appearance the device shown in Fig. 3 (b $)^{9)}$. This is one of the earliest attempt at eliminat- 


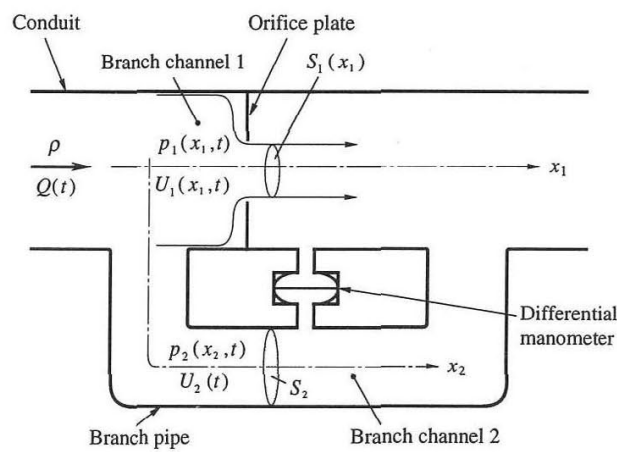

(a)

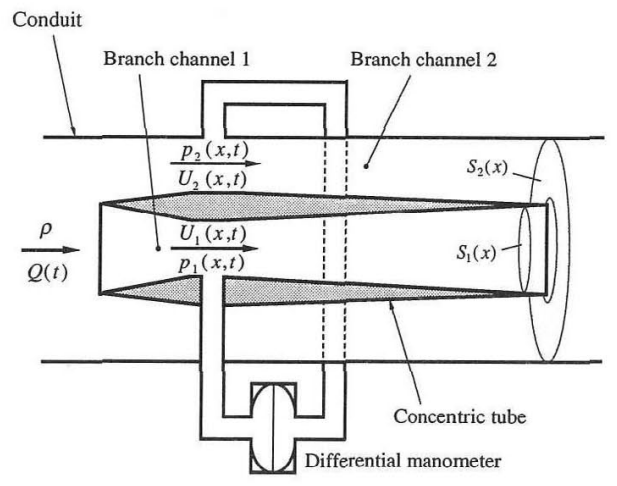

(b)

Fig. 3 Conceptual diagram of possible embodiments of the TDPF

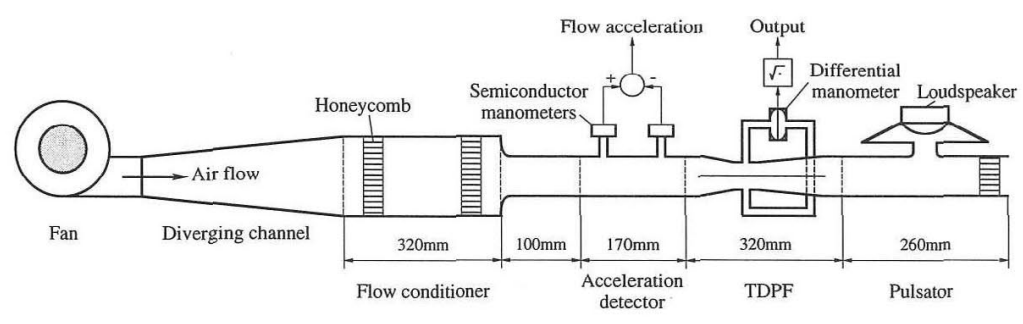

Fig. 4 Schematic layout of the experimental apparatus

ing the acceleration error, and is probably the first proposal of a flowmeter in which the pressure difference is detected across the stream. In his sensor, one of the branch streams is accelerated by the converging inner tube wall, while the remaining stream's velocity is reduced in the opposing divergent wall, and the pressure difference is detected across the inner tube wall. Although he says the pressure difference is free from the acceleration term, his arguments are qualitative and the reason is consequently not convincing. Just because the pressure difference is detected across the stream, as we have seen, it does not follow that this pressure difference does not contain the flow acceleration term. In fact, since his branch channels cannot satisfy equations (8) and (9) the pressure difference necessarily contains the acceleration term. One may say that the TDPF is the development of Randall's primitive idea into a new theory.

\section{Experiments}

An experimental apparatus was built on a trial basis. Several experiments were performed with this apparatus for air flow. Fig. 4 shows the schematic layout of the apparatus. It consists of a fan, a honeycomb flow conditioner, a flowing pipe of $20 \times 40$ $\mathrm{mm}^{2}$ rectangular cross-sectional area, a loudspeaker employed for applying pulsations, a flow acceleration detector and a TDPF branch section. The dimensions of the tested branch section are shown in Fig. 5. The pressure difference between the branch channels was detected by a variable inductance type differential manometer. In order to detect the flow acceleration, two pressure taps are made on the flowing pipe a small distance apart along the stream ${ }^{11}$. The pressure difference between these taps, which is a finite difference approximation for the axial pressure gradient and proportional to the flow acceleration, is detected by two semiconductor type manometers.

First, a steady flow was measured by the test TDPF device. The results are shown in Fig. 6. The output of the differential manometer was squarerooted and plotted as the output of the TDPF against the flow rate. The abscissa is the average flow 


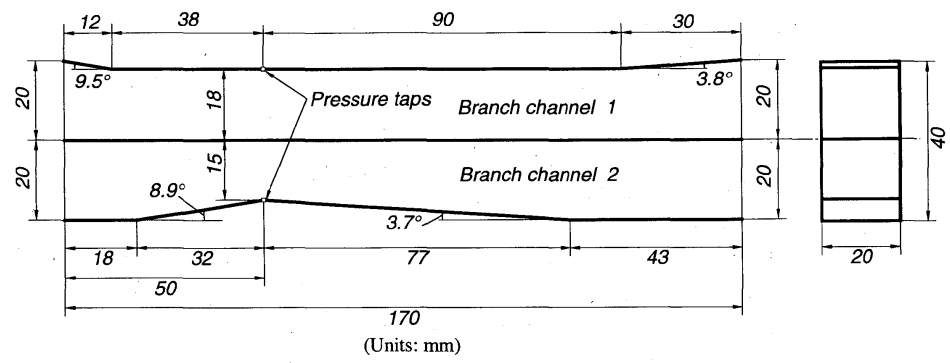

Fig. 5 Branch channels dimensions

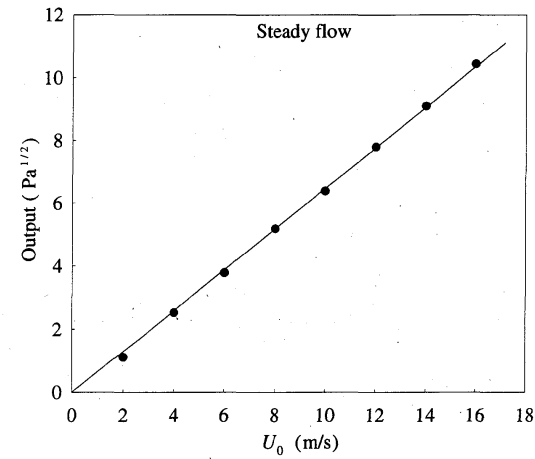

Fig. 6 Relationship between the output of the test TDPF and the flow rate for a steady flow. The ordinate is the square-rooted output of the differential manometer. The abscissa is the average flow velocity measured by a Pitot tube and a hot-wire anemometer

velocity which was calculated from the output of a Pitot tube and the velocity profile measured by a hot-wire anemometer.

Results of pulsating flow measurement are shown in Fig. 7. A sinusoidal pulsation $20 \mathrm{~Hz}$ in frequency and $0.5 \mathrm{~m} / \mathrm{s}$ in amplitude was superimposed by the loudspeaker on the otherwise steady flow. The output of the differential manometer was square-rooted and then time-averaged. The abscissa is the timeaveraged flow velocity. Fig. 6 and Fig. 7 indicate that the time-averaged rate of flow can be measured by the test TDPF device, regardless of whether the flow is pulsating or steady. Results similar to Fig. 7 were obtained for various pulsations ranging from 10 to 30 $\mathrm{Hz}$ in frequency and from 0 to $0.7 \mathrm{~m} / \mathrm{s}$ in amplitude.

Fig. 8 shows the waveform of the following signals: (a) the instantaneous flow rate, which is calculated by adding the time-averaged flow rate and the time integral of the flow acceleration; (b) the flow

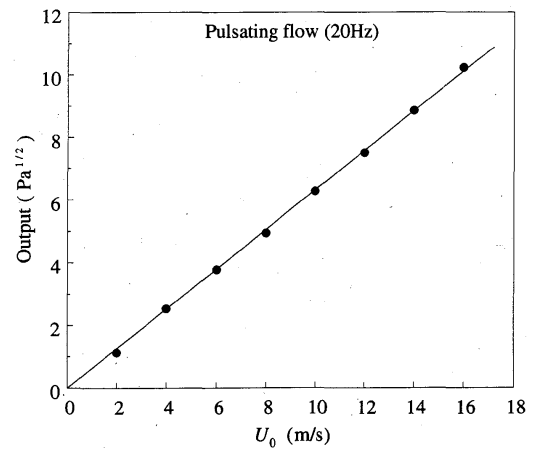

Fig. 7 Relationship between the output and the flow rate for a pulsating flow $(20 \mathrm{~Hz}$ in frequency and 0.5 $\mathrm{m} / \mathrm{s}$ in amplitude). The output of the differential manometer was square-rooted and then timeaveraged. The abscissa is the flow velocity which was averaged over time and the cross-section

acceleration detected by the two semiconductor manometers; (c) the (square-rooted) output of the test TDPF; (d) the pressure difference detected between taps upstream and downstream of the contraction - a substitute for the output of conventional differential pressure flowmeters. The time-averaged flow velocity was $2.0 \mathrm{~m} / \mathrm{s}$, and a pulsation $20 \mathrm{~Hz}$ in frequency and $0.4 \mathrm{~m} / \mathrm{s}$ in amplitude was superimposed. High frequency flow noise in each waveform was reduced by applying the multiple time averaging technique. For conventional differential pressure flowmeters, the output waveform differs from the square of the instantaneous flow rate. Further, as is seen in Fig. 8(d), although the flow never reverses, the output can become negative owing to the acceleration term. In contrast, the output of the TDPF [Fig. 8(c)] does not contain the acceleration term [Fig. 8 (b) ] and is similar to the instantaneous flow rate [Fig. 8(a)]. 


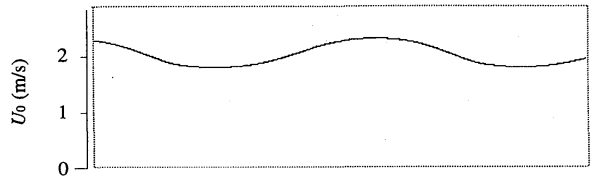

(a)

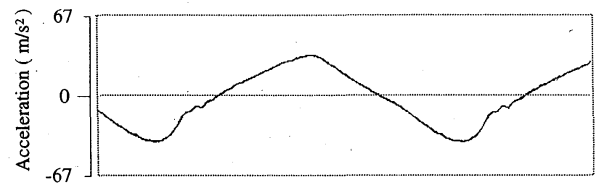

(b)

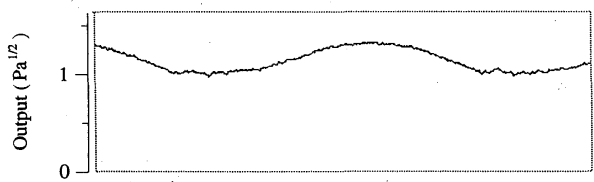

(c)

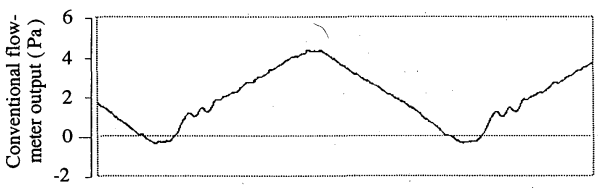

(d)

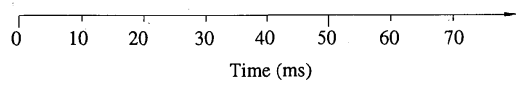

Fig. 8 Waveform of (a) the instantaneous flow rate; (b) the flow acceleration; (c) the square-rooted output of the TDPF ; (d) the output of a conventional differential pressure flowmeter

\section{Concluding Remarks}

A new method, named Transverse Differential Pressure Flowmeter, was proposed for measuring the pulsating flow rate. A trial apparatus was built and several experiments were performed for sinusoidally pulsating air flow at mean flowrates up to $16 \mathrm{~m} / \mathrm{s}$. The pulsation ranges from 10 to $30 \mathrm{~Hz}$ in frequency and from 0 to $0.7 \mathrm{~m} / \mathrm{s}$ in velocity amplitude. The uncertainty $\left(\mathrm{U}_{\mathrm{RSS}}{ }^{12)}\right)$ in the mean flowrate and the pulsation amplitude is of the order of $3 \%$. The uncertainty of calibration $\left(e^{\prime}, c^{13)}\right.$ in Fig. 6 and Fig. 7) ranges between $1 \%$ and $7 \%$. The experimental results show the following: (1) the proposed meter is capable of measuring the rate of steady flow ; (2) it is also capable of metering the time-averaged rate of pulsating flow; (3) further, it is capable of measuring the instantaneous rate of pulsating flow.

Because of the precursory nature of this study, we have concentrated on the theoretical aspects of the proposed method, and the experiments presented above are far from complete. Due to the test facility limitations, the flowing conditions are restricted within a narrow range. Future work in this study must include (1) the development of a reference standard of the pulsating flow rate; (2) the design of constructions for practical applications; (3) tests in the extended range of flow conditions; (4) tests on the effect of influence quantities ; (5) the assessment of the overall uncertainty in the measurement.

We are indebted to Mr. SHIRATA Masatoshi of the Self-Defense Force and Mr. SATOH Kenki of Civil Aviation College for their assistance in performing the experiments. We would like to express our gratitude also to Professor SADATOMI Michio of Kumamoto University and Mr. WATANABE Isao of the University of Tokyo for their helpful suggestions on the experimental apparatus.

\section{References}

1) W. M. Venable: Method of Measuring Discharge and Head, Trans. ASCE, 54-D, 502/504 (1905)

2) A. K. Oppenheim and E. G. Chilton: Pulsating-Flow Measurement - A Literature Survey, Trans. ASME, 77-2, 231/248 (1955)

3) R. W. Miller: Flow Measurement Engineering Handbook (second edition), 5-48/5-58, McGraw-Hill (1989)

4) K. Takahashi, Y. Yamashita and R. Yamaguchi : The Measurement of Laminar Pulsating Instantaneous Flow Rate with a Venturi Meter (in Japanese), Trans. SICE, 18-6, 589/595 (1982)

5) . M. Uchiyama and K. Hakomori : Measurement of Instantaneous Flow Rate. Through Estimation of Velocity Profiles, IEEE Trans. on Automatic Control, AC-28-3, 380/388 (1983)

6) K. Nakano and S. Yokota : Unsteady Flowrate Sensor by Making Use of Dynamic Characteristics of Cylindrical Chokes, IMEKO Congress, Houston (1988)

7) T. Zhao et al.: A Real Time Measuring Method of Unsteady Flow Rate and Velocity Employing a Differential Pressure in a Pipe (in Japanese), Trans. JSME (B), 52-480, 2851/2859 (1986)

8) M. Horiuchi and T. Fsobe: Dynamic Measurement of a Particular Type of Pulsating Air Flow by Making Use of an Orifice Meter (in Japanese), Trans. SICE, 28-5, 537/546 (1992)

9) L. N. Randall : Some Experimental Results in Precise Measurement of Unsteady Fluid Flow Using a Unique Flow Sensor, Flow Measurement Symposium, ASME, 205/214 (1966)

10) S. Matsunaga et al. : Contraction Type Flowmeter for 
Measuring Instantaneous Rate of Pulsating Water Flow (in Japanese), Trans. JSME (B), 45-396, 1136/ 1144 (1979)

11) I. Torigoe: A New Method for Measuring Flow Acceleration and Its Application to Pulsating Flow Measurement (in Japanese), Trans. SICE, 23-8, 794/ 799 (1987)

12) ISO/DIS 5168 : Measurement of fluid flow - Evaluation of uncertainties (1990)

13) ISO/DIS 7066/1: Assessment of uncertainty in calibration and use of flow measurement devices Part 1: Linear calibration relationships(1984)

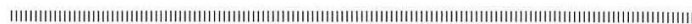
Ippei TORIGOE (Member)

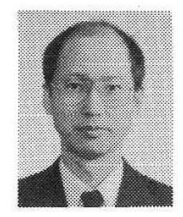

He received the B. E. degree in 1977, the M. E. degree in 1979, and the Dr. Eng. degree in 1989, all from the University of Tokyo, Tokyo, Japan. From 1980 to 1990 he worked at the University of Tokyo. Since 1990 he has been an associate professor of the Information Processing Center at Kumamoto University. His research interests are in the area of sensor engineering. 